\title{
Thyroid Care Collaborative: an electronic health record facilitating multidisciplinary management of thyroid cancer
}

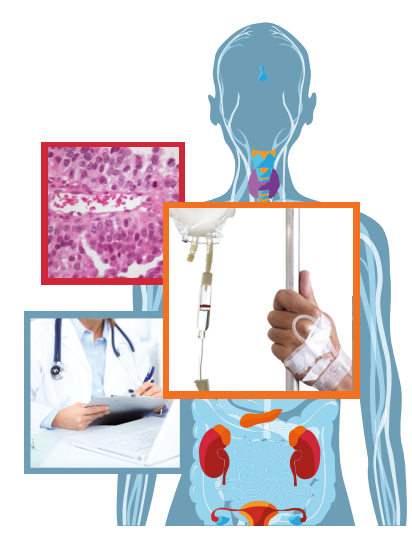

International Journal of Endocrine Oncology

\author{
Kimberly Kiplagat ${ }^{1}$, Martha J Griffin'1, Fred Baik¹,2,3, Abraham D Minkowitz ${ }^{1}$ \& Mark L \\ Urken*,1,2,3 \\ ${ }^{1}$ Thyroid, Head \& Neck Cancer (THANC) Foundation, New York, NY 10003, USA \\ ${ }^{2}$ Department of Otolaryngology - Head \& Neck Surgery, Mount Sinai Beth Israel, New York, NY 10003, USA \\ ${ }^{3}$ Department of Otolaryngology - Head \& Neck Surgery, Icahn School of Medicine, New York, NY 10029, USA \\ *Author for correspondence: mark.urken@mountsinai.org
}

\begin{abstract}
“As a patient-based, disease-specific EHR, the TCC connects all physicians involved in a patient's care by giving them direct access to the most up-to-date records, once the patient invites them to participate in the TCC. This innovative approach puts the patient at the center, and allows physicians in the care team to communicate regardless of hospital affiliation."
\end{abstract}

First draft submitted: 23 October 2017; Accepted for publication: 8 January 2018; Published online: 30 January 2018

Keywords: cancer • oncology • thyroid

In the 1990s, the concept of the electronic health record (EHR) was established to support, maintain and improve the quality of our healthcare system [1]. Since its advent, the content, structure and technology of an EHR has evolved over time, however, the goals of the EHR have remained relatively steadfast [1]. EHRs aim to improve quality of care by increasing the accessibility and comprehensiveness of health information, providing portability of health records, enhancing connectivity between physicians involved in the care of a patient, encouraging patient engagement and facilitating multidisciplinary care management. However, EHRs may serve a variety of purposes aside from patient care, such as achieving certain levels of documentation to support levels of billing for encounters. The latter often leads to the entry of endless details that are not truly relevant to a patient's disease, making it difficult and cumbersome to decipher the specific information required to provide quality care.

Specialization of web-based health-record systems have led to disease-specific databases, such as the Thyroid Care Collaborative (TCC), formerly known as the Thyroid Cancer Care Collaborative. The TCC is a thyroidspecific database that can be easily integrated into the physician workflow to improve the quality of care delivered to patients with thyroid nodules and thyroid cancer. Web-based disease-specific electronic records, such as the TCC, are dynamic and can be readily modified as new information becomes available. The following examples demonstrate the importance of this feature: the ability to add new data points such as genetic mutations that are found to influence disease virulence and patient outcomes, such as the TERT mutation; and the ability to change disease staging based on AJCC modifications or refinement of the American Thyroid Association's risk of recurrence stratification [2,3]. As new disease-specific information becomes available, the web-based EHR can evolve accordingly. The successful treatment and surveillance of thyroid cancer does not start and end with one surgeon or one endocrinologist, but rather most patients are treated in a collaborative and multidisciplinary fashion. Often those providers involved in the care of a particular patient are not in the same institution and commonly do not use the same EHR. In this paper, we will review how the TCC improves the multidisciplinary management of thyroid cancer by: enhancing communication between physicians; providing a platform for active surveillance in patients with low-risk thyroid cancers; establishing electronic feedback loops that inform the providers as to the results of further testing as well as the clinical course of that patient; implementing imaging modules that help to standardize the performance and the reporting of imaging results; delivery of specific clinical practice guidelines (CPGs) to the clinicians and the patient that are specific to where they are in their thyroid journey, to ensure adherence and 
standardization of care; providing relevant and disease-specific data that facilitates clinical research studies; and storing patient health records in one central location accessible to all members of a patient's care team.

\section{Communication between physicians}

Comprehensive thyroid-nodule and thyroid-cancer management requires collaboration between a team of specialists, which include endocrinologists, nuclear medicine physicians, pathologists, surgeons, radiologists and primary care physicians. However, communication between this wide range of providers can be fragmented and incomplete. Physicians often rely on faxed records, telephone calls and commonly, patients to bring medical records at every office visit. These archaic methods are accompanied by fragmented medical histories, delay in communication and missing or unobtainable records, often leading to delays in care and the necessity to repeat costly studies.

Despite technological advancements, there has been limited progress to provide a HIPAA-compliant system that would allow physicians who provide care to a specific patient to communicate easily and effectively. As a patientbased, disease-specific EHR, the TCC connects all physicians involved in a patient's care by giving them direct access to the most up-to-date records, once the patient invites them to participate in the TCC. This innovative approach puts the patient at the center, and allows physicians in the care team to communicate regardless of hospital affiliation. By doing so, the phenomenon of 'clinical silos' or disjointed communication of relevant health information between clinicians is eliminated [4].

Additionally, the collaborative nature of this relational database promotes the active follow-up of patients [5]. In particular, since thyroid cancer patients are at risk of developing recurrent disease, decades after their initial care is rendered, it is imperative that they be followed for life [6]. It is not uncommon that patients become complacent and forget or choose not to follow up with their clinicians. This is particularly true of younger patients who may move to different locations and have to find new doctors to be involved in their management team. Furthermore, there are certain features that are unique to the TCC and include a 'physician banner' and the 'thyroid timeline' which both help to streamline the retrieval of patient information that is readily available when a patient's record is opened.

Patients enroll in the TCC after an informed consent process, where patients learn about what the TCC has to offer, and how they can become active players in thyroid cancer research by allowing their deidentified data to be accessible for clinical research studies. Additionally, patients are informed of the wealth of resources available to them including educational videos, frequently asked questions and the ability to upload their own medical records in the patient portal. Patients are informed that the TCC is HIPAA-compliant, and meets the strictest standards for data encryption at the server and browser level.

\section{Adherence to CPGs}

Standardized management and adherence to national clinical care guidelines are important factors in the delivery of the highest quality of multidisciplinary care. Currently in the USA, standardization in thyroid cancer management is suboptimal. There is a tremendous amount of variability between treatment sites and between physicians with respect to a multitude of thyroid care decisions: the use of radioactive iodine therapy; the offering of active surveillance for patients with low risk cancers; the decision to biopsy patients with nodules that are low risk or less than a centimeter in size; and the extent of thyroid surgery and the interpretation of risk factors, such as the presence of positive central compartment lymph nodes [7].

CPGs are intended to provide clinicians with actionable clinical recommendations based on the best current evidence [7]. The American Thyroid Association (ATA) provides CPGs that include specific recommendations for the aforementioned issues. However, Haymart et al. found significant variation in the adoption of the ATA CPGs across several treatment sites. To counteract nonadherence, the TCC integrates patient data into clinical decision making modules that are based on the most recent ATA CPGs [8]. The clinical decision making modules provide the clinician with a suggested treatment plan unique to the specific patient in question. In doing so, patients can be assured that they are receiving care upheld to the national standard and clinicians can remain diligent in their treatment plan regardless of specialty or hospital affiliation.

\section{Electronic feedback loops}

In order to keep pace with the ever-evolving national guidelines and body of scientific knowledge, healthcare providers and information technology professionals must work in partnership to generate methods of continual self assessment through electronic feedback loops. The TCC has an established feedback loop system that aids 
in self-monitored modification and fine-tuning of diagnostic and therapeutic interventions [4]. By enhancing communication of physicians involved in a patient's care, all the care team members can remain informed regarding the health status and the clinical course of their shared thyroid cancer patient.

The TCC eliminates 'clinical silos', or the disjointed transference of relevant health information between clinicians involved in a patient's care [4] through a notification system. Automated emails are sent to the approved members of the care team each time the patient undergoes a new treatment, encounter or diagnostic work up. In a recent clinical commentary, Urken et al. describes a clinical vignette in which a surgical pathologist identifies a discrepancy between the final diagnosis post-thyroidectomy and the initial interpretation of the disease by a cytopathologist [4]. The surgical pathologist utilizes the TCC to seamlessly notify the original cytopathologist, encouraging the review of the initial interpretation. Other incidences where feedback loops can be very useful in the diagnostic evaluation of patients with thyroid cancer relates to the assessment of suspicious lymph nodes in the lateral compartment of the neck. It is common that the individuals performing those ultrasounds never receive feedback as to what was ultimately found in the final pathology. A built-in system for automatic feedback to clinicians helps to promote continuous quality improvement in a multidisciplinary setting.

\section{Disease maps}

In addition to electronic feedback loops, the TCC encourages the use of 'disease mapping' to ameliorate problems arising from inadequate disease-specific communication between physicians. With the help of a user-friendly imaging module, a physician can create a visual thyroid map of specific nodules and lymph nodes, with their imaging and cytologic characteristics attached. This visual map can enhance the reporting of ultrasound, cytologic, surgical and pathologic details obtained during the treatment of a patient. By disseminating this accurate, portable and real time information to the patient's care team, disease understanding and treatment accuracy is improved. By creating a disease map that follows a patient on their clinical journey, the 'handoffs of care' that take place between clinicians can be done more accurately and safely. Specifically, the disease-mapping modules aim to improve the accuracy of ultrasound and cytologic reporting, lymph node mapping, nodule tracking, surgical planning, pathologic reporting and post-treatment surveillance.

\section{Active surveillance}

The TCC provides a platform for meticulous disease surveillance. Thyroid cancer incidence rates have increased threefold in the USA between 1975 and 2009, primarily through increased detection of papillary thyroid cancers that are not clinically evident [9]. According to the SEER program, detection of tumors $<1 \mathrm{~cm}$ has dramatically increased from 25\% (1988-1989) to 39\% (2008-2009) of the number of patients diagnosed with thyroid cancer [9]. The ATA modified the 2015 guidelines in response to these data and advised clinicians that properly selected patients with low-risk thyroid cancer may be managed expectantly and not undergo immediate surgery [2].

Active surveillance is a thyroid cancer-management strategy that entails observation using ultrasonography to monitor disease progression for low-risk thyroid cancer [10]. Preliminary studies on active surveillance of thyroid cancer have yielded promising results. Ito $e t$ al. found an increase in tumor size for $6.4 \%$ of 340 patients who underwent active surveillance for low-risk thyroid cancer at the 5-year follow-up and $15.9 \%$ of 340 patients had tumor enlargement at the 10-year follow-up [11]. A total of 109 of the 340 patients who selected the active surveillance route underwent surgery for a variety of reasons, none of whom showed cancer recurrence [11]. Further research is required to firmly establish active surveillance as a viable management approach for low-risk thyroid cancer.

The TCC is a potentially powerful tool for implementing active surveillance as a treatment strategy for papillary thyroid microcarcinomas. For patients who have chosen active surveillance, the TCC provides the technology for physicians to monitor their patients' progress and to capture data that would be essential for long-term follow-up. The imaging and cytology module in the TCC records up to 33 ultrasound parameters for thyroid nodules and cervical lymph nodes [10]. By capturing important data points, the TCC is an effective vehicle to follow patients long-term and can act as a safety net to prevent lost follow-ups. Communication between physicians is also enhanced since all the physicians in the care team have access to the patients' medical reports. Brito et al. discussed characteristics required for patients to be eligible for active surveillance, including, patient age, tumor size, location and willingness to comply with lifelong follow-up [12]. These factors are already captured by the TCC.

Since active surveillance management would require patients to be followed up throughout their life, the TCC aims to encourage patient involvement in their own care. The TCC provides patients with a portal where they can 
access personal medical records, an extensive educational video library, and thyroid-related medical information. These resources can be accessed wherever there is internet. By engaging patients, the TCC can be an important vehicle to improve the high rates of patients who are lost to follow-up in the USA, and ensuring that patients who are enrolled in active surveillance adhere to the protocol given [13].

\section{Clinical trials}

Another strength of creating and supporting a comprehensive clinical registry is the ability to seamlessly and efficiently enroll patients in clinical trials. In addition to serving as a supplementary resource to large-scale institutional registries, the TCC can also serve as a directory of potential clinical trial opportunities. Care team members can use this TCC to direct their individual patients toward specific trials given their patient's unique health status [5]. Clinical trial opportunities can be disseminated and implemented in two ways, one passive and one active.

The passive method allows clinical trials to employ the HIPAA-compliant query function of the database to identify patients based on a trial's unique set of inclusionary and exclusionary criteria. Once the list of eligible patients is generated, the TCC can be programmed to reach out to the members of each patient's care team to inform clinicians of clinical trial availability. It would then be left to the expert discretion of the care team whether the patient would benefit from such an opportunity.

In the case of large-scale interest from several clinical trial coordinators, the TCC could implement the active option. The active method would involve programming the inclusionary and exclusionary criteria of the approved clinical trials into the TCC. An automated email would then notify care team members of candidacy for clinical trials. Once they are alerted to an existing trial or trials, it would be left up to the qualified healthcare providers to discuss enrollment with the patient.

\section{Disease \& research-specific registries}

Health registries play a powerful role in utilizing clinical data with the aim of advancing research and outcomes, improving quality of care and understanding disease processes [5]. Cancer registries such as the Surveillance, Epidemiology and End Results (SEER) program are important databases that provide meaningful information to clinicians and researchers. However, because cancer registries process data for all types of cancer and need to remain up to date as to a patient's clinical course, these registries are necessarily limited vehicles for high quality research [5].

Mehra et al. reviewed population-based cancer registries, health systems-based registries and patient-based registries to elucidate their efficacy in cancer research [5]. According to their analysis, population-based cancer registries and health systems-based registries are effective in demonstrating the following: trends in cancer incidence nationally; survival status and cause of death data; and elucidating the geographical data with respect to new cancer cases. However, several limitations persist [5]. First, active follow-up for specific variables is unavailable in these registries. Second, follow-up for surveillance and/or treatment in different health systems is difficult. Third, data entry does not occur at point-of-care allowing for discrepancies. Finally, case reporting and data analysis are not timely [5]. In the next section, we discuss the role of the TCC in addressing these shortcomings.

\section{Thyroid cancer registry}

Management of thyroid cancer often relies heavily on experts due to the lack of high-quality data [14-16]. For example, for patients with differentiated thyroid cancer, there is a lack of agreement among clinicians and researchers concerning extent of surgery, postoperative treatment and surveillance recommendations [5]. Additionally, understanding the role of genetics in thyroid cancer and pathological features of lymph nodes requires a large data set and detailed analysis [5]. To address the shortcomings of current cancer registries, the TCC provides a wealth of unique disease-specific data which can be used to advance thyroid cancer research.

By collecting dynamic data fields, integrating data entry into a physician's workflow, enabling patient-driven lifetime follow-up for treatments, diagnostic tests, etc. irrespective of health system, representing an entire spectrum of disease, and providing a comparative lens by which physicians can measure their data against national trends, the TCC overcomes the shortcomings of current registries [5].

\section{Interoperability}

A limitation of the TCC is the lack of integration into providers' EHRs, which is an area in active development. An important solution for facilitating integration of TCC to other providers' EHRs include opportunities to 'cut and paste' data recorded in the TCC to other EHRs. Second, auto-population of pathology reports and laboratory 
reports to other EHRs is another way to enhance record keeping [5]. Interoperability refers to the extent to which systems and devices can exchange data and interpret that shared data [17]. This is a major obstacle facing the EHR system in the USA. The TCC aims to bridge the gaps between hospital affiliation and EHR, thus making critical health information available to all members of an individual's care team. Geographic universality is accomplished by storing all the patient health information on a cloud server which can be accessed anywhere and at any time. Upon registration, a unique identification number is assigned to each patient, allowing for ease of HIPAA-compliant data retrieval. The identification number also allows for other care team or research team members to access the information despite their physical location. Additionally, the interoperability of the TCC does not only benefit the doctor, but the patient as well. In the case of a new referral or even a cross country move, the cloud server feature allows the patient to transition seamlessly between medical practices while maintaining control of all their current health records.

\section{Conclusion}

The TCC is a powerful web-based tool for facilitating management of thyroid nodules and thyroid cancer by enhancing communication between physicians, ensuring adherence to clinical guidelines, generating electronic feedback loops for quality care, improving physicians' reporting of imaging details through disease maps, providing a platform for following active surveillance candidates, serving as a disease specific platform for clinical trials and acting as an effective disease-specific registry for thyroid cancer. In this discussion, we demonstrate that by transforming the way physicians communicate with each other and with patients, we can make significant strides in healthcare quality and delivery.

\section{Acknowledgements}

The authors wish to thank the Mount Sinai Health System for research support.

Financial \& competing interests disclosure

The authors would like to acknowledge the generous support of this research by the Mount Sinai Health System and the Thyroid, Head and Neck Cancer (THANC) Foundation. ML Urken is the medical advisor of the THANC Foundation. The authors have no other relevant affiliations or financial involvement with any organization or entity with a financial interest in or financial conflict with the subject matter or materials discussed in the manuscript apart from those disclosed.

No writing assistance was utilized in the production of this manuscript.

\section{Open access}

This work is licensed under the Creative Commons Attribution 4.0 License. To view a copy of this license, visit http://creativecomm ons.org/licenses/by/4.0/.

\section{References}

1. Hoerbst A, Ammenwerth E. Electronic health records. A systematic review on quality requirements. Methods Inf. Med. 49(4), 320-336 (2010).

2. Haugen BR. 2015 American Thyroid Association Management Guidelines for Adult Patients with Thyroid Nodules and Differentiated Thyroid Cancer: what is new and what has changed? Cancer 123(3), 372-381 (2017).

3. Suh S, Kim YH, Goh TS et al. Outcome prediction with the revised American joint committee on cancer staging system and American thyroid association guidelines for thyroid cancer. Endocrine 58(3), 495-502 (2017).

4. Urken ML, Randolph G, Orloff L et al. The role of electronic feedback loops in the continuous quality improvement of thyroid nodule and thyroid cancer care. Endocr. Pract. 23(8), 1024-1027 (2017).

5. Mehra S, Tuttle RM, Milas M et al. Database and registry research in thyroid cancer: striving for a new and improved national thyroid cancer database. Thyroid 25(2), 157-168 (2015).

6. Hedman C, Strang P, Djarv T, Widberg I, Lundgren CI. Anxiety and fear of recurrence despite a good prognosis: an interview study with differentiated thyroid cancer patients. Thyroid 27(11), 1417-1423 (2017).

7. Likhterov I, Tuttle RM, Haser GC et al. Improving the adoption of thyroid cancer clinical practice guidelines. Laryngoscope 126(11), 2640-2645 (2016).

8. Stack BC Jr, Ferris RL, Goldenberg D et al. American Thyroid Association consensus review and statement regarding the anatomy, terminology, and rationale for lateral neck dissection in differentiated thyroid cancer. Thyroid 22(5), 501-508 (2012).

9. Davies L, Welch HG. Current thyroid cancer trends in the United States. JAMA Otolaryngol. Head Neck Surg. 140(4), 317-322 (2014). 
10. Haser GC, Tuttle RM, Su HK et al. Active surveillance for papillary thyroid microcarcinoma: new challenges and opportunities for the health care system. Endocr. Pract. 22(5), 602-611 (2016).

11. Ito $\mathrm{Y}$, Miyauchi A, Inoue $\mathrm{H}$ et al. An observational trial for papillary thyroid microcarcinoma in Japanese patients. World J. Surg. 34(1), 28-35 (2010).

12. Brito JP, Ito Y, Miyauchi A, Tuttle RM. A clinical framework to facilitate risk stratification when considering an active surveillance alternative to immediate biopsy and surgery in papillary microcarcinoma. Thyroid 26(1), 144-149 (2016).

13. Johnson CJ, Weir HK, Yin D, Niu X. The impact of patient follow-up on population-based survival rates. J. Registry Manag. 37(3), 86-103 (2010).

14. National Comprehensive Cancer Network (NCCN). Clinical Practice Guidelines in Oncology: Thyroid Carcinoma (2013).

15. Cobin RH, Gharib H, Bergman DA et al. AACE/AAES medical/surgical guidelines for clinical practice: management of thyroid carcinoma. American Association of Clinical Endocrinologists. American College of Endocrinology. Endocr. Pract. 7(3), 202-220 (2001).

16. American Thyroid Association Guidelines Taskforce on Thyroid N, Differentiated Thyroid C, Cooper DS et al. Revised American Thyroid Association management guidelines for patients with thyroid nodules and differentiated thyroid cancer. Thyroid 19(11), 1167-1214 (2009).

17. O'Connor $S$. What is interoperbaility, and why is it important (2017).

https://healthcare.adsc.com/blog/what-is-interoperability-and-why-is-it-important 